\title{
Novel plasma exosome biomarkers for prostate cancer progression in co-morbid metabolic disease
}

Naser Jafari ${ }^{1}$, Manohar Kolla ${ }^{1}$, Isabella R. Pompa ${ }^{1}$, Yuhan Qiu ${ }^{1}$, Christina S. Ennis ${ }^{1}$, Joakin Mori ${ }^{2}$, Kiana Mahdaviani $^{2}$, Meredith Halpin ${ }^{2}$, Gretchen A. Gignac ${ }^{2}$, Christopher M. Heaphy ${ }^{2}$, Gerald V. Denis ${ }^{1,2,3,4 *}$

${ }^{1}$ Boston University-Boston Medical Center Cancer Center, Boston University School of Medicine, Boston, MA 02118, USA

${ }^{2}$ Section of Hematology and Medical Oncology, Department of Medicine, Boston University School of Medicine and Boston Medical Center, Boston, MA 02118, USA

${ }^{3}$ Department of Pharmacology and Experimental Therapeutics, Boston University School of Medicine, Boston, MA 02118, USA

${ }^{4}$ Shipley Prostate Cancer Research Professor, Boston University School of Medicine, Boston, MA 02118, USA

*Corresponding author. E-mail: gdenis@bu.edu

Running title: BRD4 regulates EMT in CRPC

Grant support: This study was supported by grants from the National Institutes of Health (DK090455, U01CA182898, R01CA222170; GV Denis).

Corresponding author: Gerald V. Denis, Boston University-Boston Medical Center Cancer Center, Rm K520, Boston University School of Medicine, 72 East Concord Street, Boston, Massachusetts, 02118, USA

Phone: 617-358-4785

Fax: 617-638-5673

E-mail: gdenis@bu.edu

Word count: 2,856 including Abstract

Abstract word count: 172

Figures: 6

Supplementary Figures: 4

Supplementary Tables: 2

References: 82 


\begin{abstract}
Comorbid Type-2 diabetes (T2D), a metabolic complication of obesity, associates with worse cancer outcomes for prostate, breast, head and neck, colorectal and several other solid tumors. However, the molecular mechanisms remain poorly understood. Exosomes as carriers of miRNAs in blood encode the metabolic status of the originating tissues and deliver their cargo to target tissues. We hypothesized that T2D plasma exosomes induce epithelial-mesenchymal transition (EMT) and immune checkpoints in prostate cancer cells. Here, we show that plasma exosomes from subjects with T2D induce EMT features in prostate cancer cells and upregulate the checkpoint genes $C D 274$ and $C D 155$. We prove that specific exosomal miRNAs abundant in T2D plasma (miR374a-5p, miR-93-5p, miR-424-5p) are delivered to tumor cells and regulate these target genes. We build on our previous reports showing BRD4 controls migration and dissemination of castration-resistant prostate cancer and transcription of key EMT genes, to show that T2D exosomes drive EMT and immune ligand expression through BRD4. The results suggest novel, non-invasive approaches to evaluate prostate cancer progression risk in patients with comorbid T2D.
\end{abstract}

172 words

Keywords: epithelial-mesenchymal transition, epithelial plasticity, prostate cancer, castrationresistant, epigenetic regulators, BET proteins, next-generation BET degrader, BRD4

Abbreviations: ADT, androgen deprivation therapy; BET, Bromodomain and ExtraTerminal; DAPI, 4',6-diamidino-2-phenylindole dihydrochloride; EMT, epithelial-to-mesenchymal transition; FBS, fetal bovine serum; FDR, false discovery rate; IFN, interferon; IPA, Ingenuity Pathway Analysis; ND, non-diabetic; PCA, principal components analysis; PD-L1, programmed death-ligand 1; PROTAC, proteolysis-targeting chimera; PSA, prostate specific antigen; T2D, Type 2 diabetes; TGF, Transforming Growth Factor; VST, variance-stabilizing transformation 


\section{Introduction}

The incidence of obesity-driven diabetes continues to increase worldwide, and parallel to this trend, an increase in incidence of several obesity-related cancers has been reported [1]. Already in the U.S., for example, $>100$ million adults have been diagnosed with diabetes or pre-diabetes [2], about $90 \%$ as Type 2 diabetes (T2D) [3], a frequent metabolic complication of obesity, emphasizing the scale of the public health challenge. The severity of comorbid T2D also predicts worse cancer outcomes; associations are well established for breast cancer [4], head and neck cancer [5], colorectal cancer [6] and several other solid tumors. In prostate [7, 8] and other cancers [9], increased prevalence of comorbidity also correlates with advanced age [10] and worse outcomes. Yet the cellular and molecular mechanisms that explain these associations remain poorly understood. Comorbidity is insufficiently considered in clinical decision making. These knowledge gaps are important for medically underserved patient populations, where obesity and T2D are prevalent $[11,12]$, often in association with food deserts and an obesogenic built environment [13]. There is urgent need for novel, robust biomarkers to evaluate risks for cancer progression and assist clinical decision making for such underserved patients [14, 15].

New work in molecular endocrinology is revealing that cancer patients with comorbid chronic obesity and/or metabolic complications have adverse signaling in the adipose microenvironments of breast [16-19] or prostate tumors [20-22], compared to patients with the same type and stage of cancer, who are otherwise metabolically normal. In prostate cancer, obesity and its metabolic complications have been studied intensively to identify serological and histological data that would help identify cases at high risk for failure of androgen deprivation therapy (ADT), progression and metastasis in patients with these co-morbid conditions. Metabolic biomarkers that include elevated insulin-like growth factor-1, insulin and C-peptide $[23,24]$, leptin, glucose $[25,26]$, pro-inflammatory cytokines, lipid profiles [27] and androgen 
levels have all been associated with worse outcomes [28, 29]. Metabolic status is also a concern because ADT is known to induce insulin resistance [30]. However, clinical trials have not yet established which serological markers of metabolism are most informative for the wide range of prostate cancer patients on various ADT and metabolic medications, and at which stages of disease progression specific markers would have greatest value. Innovative directions would be helpful.

We took as a starting point the clinical observation that poor control of T2D in prostate cancer associates with rapid emergence of resistance to the anti-androgens abiraterone acetate and enzalutamide, compared to prostate cancer patients with well controlled blood glucose [26]. Noninvasive biomarkers have been explored for diagnostic, prognostic and therapeutic utility, including most recently circulating tumor DNA [31] and microRNAs (miRNA) [32]. In particular, miRNA biomarkers have gained attention because, unlike other nucleic acid biomarkers, these factors may be functional in prostate cancer [33]. Upon delivery to target tissues, miRNAs are capable of reprogramming cell metabolism and fate to affect the course of progression, metastasis and therapeutic responses. Deeper understanding of miRNA mechanism and gene targets may suggest novel therapeutics or prognostic biomarkers [34] to understand progression risks. We wondered whether circulating miRNAs might differ between T2D and non-diabetic (ND) subjects.

Significant evidence implicates exosomes as carriers of miRNAs in blood [35], saliva [36] or other body fluids that can be sampled noninvasively for biomarker assessment in cancer. Several studies have investigated blood miRNAs derived from tumors as an approach to evaluate cancer diagnosis [37, 38], prognosis [39] and recurrence [40]. However, we took a converse approach, and investigated plasma exosomal miRNAs as biomarkers of co-morbid T2D that instead might have functional impact on prostate cancer progression. Our rationale is that obesity-driven 
metabolic disease has long been studied in prostate cancer incidence [41, 42], progression [43] and prostate cancer-specific mortality [44], although diabetes has been shown to be protective in some prostate cancer studies $[45,46]$. Despite intensive investigation, the mechanisms and clinical variables most strongly associated with incidence, progression, distant metastasis and mortality [41, 47], and the impact of metabolic medications [48], have not been settled. We considered that plasma exosomes in subjects with obesity-driven T2D might be leveraged to assess risk of progression and treatment resistance in prostate cancer, and might have functional significance to understand mechanisms of tumor progression.

We recently showed that the metabolic status of mature adipocytes determines the payload of released exosomes. Adipocytes that have been rendered insulin resistant by exposure to proinflammatory cytokines, or that were isolated from adipose tissue of adult subjects with T2D, release exosomes that drive increased migration, invasiveness, epithelial-to-mesenchymal transition (EMT), gene expression associated with cancer stem-like cell formation and aggressive, pro-metastatic behavior in breast cancer cell models, compared to adipocytes that are insulin sensitive or isolated from adipose tissue of ND subjects [49]. We built on these observations and hypothesized that similar phenotypes would be observed for plasma exosomes from T2D subjects compared to ND controls, using prostate cancer cell lines as a readout.

Here, we report that exosomes from peripheral blood plasma of subjects with T2D induce EMT in DU145 cells, a model for hormone-refractory and aggressive prostate cancer. Interestingly, exosomes purified from the plasma of ND subjects suppressed transcription of classical EMT genes in the same model, suggesting that ND exosomes may harbor cancer chemopreventive miRNAs. We were surprised to find in the same system that T2D exosomes also induce expression of the immune checkpoint gene $C D 274$, which encodes the immune checkpoint protein PD-L1. We had previously shown that the somatic members of the 
Bromodomain and ExtraTerminal (BET) family of proteins (BRD2, BRD3, BRD4) are positive co-regulators of the PD-1/PD-L1 axis in triple negative breast cancer models [50]. We have also shown that BRD4 regulates migration and dissemination of castration-resistant prostate cancer and transcription of key EMT genes [51, 52]. Using the pan-BET inhibitor JQ1 and the BRD4selective PROTAC degrader MZ-1, we further demonstrate here that BRD4 is a critical effector for plasma exosome-driven prostate cancer aggressiveness, and functionally couples EMT and immune checkpoint gene expression in prostate cancer. Our results lay the groundwork for a deeper, clinical translational investigation of plasma exosomes as functionally critical drivers of prostate tumor progression in patients with comorbid T2D, and as potential biomarkers that are both robust and non-invasive. 


\section{Materials and Methods}

\subsection{Cell lines and reagents}

Cell lines were previously described $[51,52]$. The DU145 prostate cancer cell line was cultured in RPMI-1640 medium (Gibco). All culture media were supplemented with 10\% fetal bovine serum (FBS, Corning) and 1\% antibiotics (penicillin/streptomycin, Gibco). As positive controls for induction of EMT genes or PD-L1, we treated the cells with transforming growth factor (TGF)- $\beta$ or interferon (IFN)- $\gamma$ (5 ng/mL), respectively, as we previously published [53]. Paraformaldehyde solution (AAJ19943K2, Thermo Scientific) and 4',6-diamidino-2phenylindole dihydrochloride (DAPI; FluoroPure grade; D21490, Thermo Scientific) were used to fix and stain the nuclei. Recombinant Human Interferon gamma protein (Active) (ab259377), and Recombinant human TGF beta 1 protein (ab50036) were purchased from Abcam.

\section{2. $\quad$ RNA staining and immunofluorescence imaging}

Exosomal RNA was stained using SYTO ${ }^{\mathrm{TM}}$ RNASelect $^{\mathrm{TM}}$ from Thermofisher (cat number: S32703) according to the manufacturer's protocol. Alexa Fluor ${ }^{\mathrm{TM}} 568$ Phalloidin (Thermofisher, A12380) was used to stain the actin fibers. Cellular nuclei were stained by DAPI, FluoroPure ${ }^{\mathrm{TM}}$ grade (Thermofisher, D21490).

\section{3. $\quad q R T-P C R$}

Procedures were as previously described $[51,52]$. Briefly, total RNA was extracted from tumor cells using an RNAeasy Kit (Qiagen). Reverse transcription reactions were performed with $1 \mu \mathrm{g}$ of total RNA with the QuantiTect Reverse Transcription kit (Qiagen). Gene expression was measured using TaqMan ${ }^{\mathrm{TM}}$ master mix (Thermofisher, 4369510) and human gene probes as follows: SNAI (Hs00195591_m1), SNA2 (Hs00950344_m1), CDH1 (Hs01023895_m1), ACTB 
(Hs00357333_g1), CD274 (encodes PD-L1, Hs01125301_m1), CD155 (encodes PVR or TIGIT ligand, Hs00197846_m1), VIM (Hs00958111_m1), TGFB1 (Hs00998133_m1), and AHNAK (Hs01102463_m1).

\subsection{PCR array}

RNA was isolated using QuantiTect Reverse Transcription Kit (Qiagen), and $1 \mu \mathrm{g}$ of each sample was used to prepare $20 \mu \mathrm{L}$ cDNA using RNeasy Plus Mini Kit (Qiagen). Human RT ${ }^{2}$ ProfilerTM PCR Array, including Epithelial to Mesenchymal Transition (EMT) genes (PAHS090Z), Cancer Stem Cell genes (PAHS-176ZC), and miRNA Array were purchased from Qiagen. Reverse transcription reactions were performed with $\mathrm{RT}^{2} \mathrm{SYBR}$ Green ROX qPCR Mastermix (Qiagen). Exosomal microRNAs were profiled using Human Serum/Plasma miRCURY LNA miRNA PCR array (Qiagen, \# YAHS-106Y, Plate Format: 2 x 96-well).

\subsection{Gene expression analysis}

All $\mathrm{C}_{\mathrm{t}}$ values of the genes were normalized to the respective $A C T B$ gene $\left(\Delta \mathrm{C}_{\mathrm{t}}\right)$. Then $\Delta \mathrm{C}_{\mathrm{t}}$ of each gene was subtracted from the control gene $\Delta \mathrm{C}_{\mathrm{t}}\left(\Delta . \Delta \mathrm{C}_{\mathrm{t}}\right)$. For the control groups $\Delta . \Delta \mathrm{C}_{\mathrm{t}}$ was calculated using this formula: $\Delta \Delta \mathrm{C}_{\mathrm{t}}=\Delta \mathrm{C}_{\mathrm{t}}(\mathrm{C} 1$ or $\mathrm{C} 2$ or $\mathrm{C} 3)-\Delta \mathrm{C}_{\mathrm{t}}$ (control average). Then, fold change was calculated using $2^{\wedge}-\Delta \Delta \mathrm{C}_{\mathrm{t}}$, $\left(2\right.$ to the power of negative $\left.\Delta \Delta \mathrm{C}_{\mathrm{t}}\right)$.

Next, the Z score was calculated based on this formula:

$\mathrm{Z}$ score $=(\mathrm{X}-\mathrm{mean}) / \mathrm{SD}$, in which $\mathrm{X}$ is the fold change. Bio Vinci software $($ San Diego, CA) was used to cluster the genes. In order to predict disease and function, data were analyzed through the use of Ingenuity Pathway Analysis, IPA (QIAGEN Inc., https:/www.qiagenbioinformatics.com/products/ingenuity-pathway-analysis). 


\subsection{Exosome isolation and characterization}

Patient whole blood was obtained commercially from Research Blood Components, LLC (Watertown, MA) on ice pack, centrifuged $\left(16 \mathrm{k} \mathrm{rpm}, 4^{\circ} \mathrm{C}, 30^{\prime}\right)$ to separate plasma, which was clarified again by centrifugation and filtration $(0.2 \mu \mathrm{m})$ to remove large vesicles or apoptotic bodies. Exosomes were purified by size exclusion using qEV columns by automatic fractionation collector. The columns were packed with spherical beads $(35 \mathrm{~nm}$ pore size $)$ such that the spaces between the pores fractionate exosomes with size range of $35 \mathrm{~nm}-150 \mathrm{~nm}$. The exosomes were eluted using PBS with $1 \mathrm{mM}$ EDTA and stored at $4{ }^{\circ} \mathrm{C}$ for exosome size distribution and concentration measurements using a NanoSight NS300 system. Exosomal preparations underwent quality control analysis as previously published [49]. T2D and ND plasma origin exosomes were normalized to $10^{9}$ particles added per cell culture well.

\subsection{MicroRNA profiling of plasma exosomes}

Exosomal RNA was isolated using exoRNeasy Midi Kit (Qiagen, 77144), For miRNAspecific first-strand cDNA synthesis, miRCURY LNA RT Kit (Qiagen, 339340) was used. Then, the PCR array analysis was conducted using miRCURY LNA SYBR Green PCR Kit (Qiagen, 339346) and Human Serum/Plasma miRCURY LNA miRNA PCR array (Qiagen, Catalog Number: YAHS-106Y, Plate Format: 2X96-Well) to detect plasma exosomes.

\section{8. microRNA delivery to DU145 cells}

Differentially expressed microRNAs in T2D exosomes were transfected using Lipofectamine RNAiMAX Transfection Reagent (13778150, Invitrogen). The human homologs of the synthesized microRNA mimics were purchased from Thermofisher. The miRBase Accession numbers are as follows: hsa-miR-374a-5p (MIMAT0000727), hsa-miR-93-5p 
(MIMAT0000093), and hsa-miR-424-5p (MIMAT0001341). After transfection, cells were incubated for $48 \mathrm{hr}$ at $37^{\circ} \mathrm{C}$, whereupon cellular total RNA was isolated and expression of SNAI1, PDL1, CDH1 and ACTB was analyzed in transfected cells.

\subsection{Flow cytometry analysis}

Single-cell suspensions were washed after collection and stained in ice-cold $\mathrm{Ca}^{+2} / \mathrm{Mg}^{+2}$-free PBS with a viability dye (Zombie NIR, BioLegend) for $20 \mathrm{~min}$ at $4{ }^{\circ} \mathrm{C}$ in the dark. Cell suspensions were then washed twice with ice-cold flow cytometry buffer $\left(\mathrm{Ca}^{+2} / \mathrm{Mg}^{+2}\right.$-free $\mathrm{PBS}$, supplemented with 2\% FBS and 2 mM EDTA). Cell suspensions were then stained for PD-L1 (PE-conjugated, BD Biosciences). All cell suspensions were washed twice in ice cold flow cytometry buffer before analysis. Unstained cells and single-stained controls were used to calculate flow cytometry compensation. Data acquisition (typically 1 million events) was performed on a BD LSRII instrument at the Boston University Flow Cytometry Core Facility. Data analysis was carried out using FlowJo Software (version 10.6.1, Tree Star).

\subsection{0. $R N A$ sequencing}

Total RNA was isolated from DU145 cells that were untreated controls or were treated with exosomes isolated from the plasma of three ND subjects or three T2D subjects. Each experimental group was represented in biological triplicate. RNA sequencing workflow was conducted by Boston University School of Medicine Microarray and Sequencing Core. FASTQ files were aligned to human genome build hg38 using STAR [54], (version 2.6.0c). EnsemblGene-level counts for non-mitochondrial genes were generated using featureCounts (Subread package, version 1.6.2) and Ensembl annotation build 100 (uniquely aligned proper pairs, same strand). Separately, SAMtools (version 1.9) was used to count reads aligning in proper pairs at 
least once to either strand of the mitochondrial chromosome (chrM) or to the sense or antisense strands of Ensembl loci of gene biotype "rRNA" or of non-mitochondrial RepeatMasker loci of class "rRNA" (as defined in the RepeatMasker track retrieved from the UCSC Table Browser). FASTQ quality was assessed using FastQC (version 0.11.7), and alignment quality was assessed using RSeQC (version 3.0.0).

Variance-stabilizing transformation (VST) was accomplished using the "Variance Stabilizing Transformation" function in the DESeq2 R package (version 1.23.10) [55]. (Principal Component Analysis (PCA) was performed using the prcomp R function with variance stabilizing transformed (VST) expression values that were z-normalized (set to a mean of zero and a standard deviation of one) across all samples within each gene. Differential expression was assessed using the likelihood ratio test and Wald test implemented in the DESeq2 R package. Correction for multiple hypothesis testing was accomplished using the Benjamini-Hochberg false discovery rate (FDR). All analyses were performed using the R environment for statistical computing (version 4.0.2).

\subsection{Statistical analysis}

To identify genes whose expression changes coordinately with respect to exosome treatment groups, a one-way analysis of variance (ANOVA) was performed using a likelihood ratio test to obtain a p value for each gene. Benjamini-Hochberg False Discovery Rate (FDR) correction was applied to obtain FDR-corrected p values (q values), which represent the probability that a given result is a false positive based on the overall distribution of $\mathrm{p}$ values. Corrected/adjusted $\mathrm{p}$ values such as the FDR q are the best measure of significance for a given test when many hypotheses (genes) are tested at once. The FDR q value was also recomputed after removing genes that did not pass the "independent filtering" step in the DESeq2 package. Genes with low overall 
expression are more strongly affected by random technical variation and more likely to produce false positive results. Wald tests were then performed for each gene between experimental groups to obtain a test statistic and $\mathrm{p}$ value for each gene. Statistical analyses of the in vitro experiments were performed using Student's $t$ test or ANOVA as indicated, and were generated by GraphPad Prism software. $p<0.05$ was considered statistically significant. 


\section{Results}

\subsection{Exosomes from plasma of T2D subjects induce EMT in prostate cancer cell lines}

We built on our previous report that exosomes from conditioned media of mature adipocytes induce transcription of EMT genes in breast cancer cell lines. This induction is more pronounced if the adipocytes are insulin resistant or obtained from the adipose tissue of subjects with T2D [49]. First, we tested whether plasma exosomes phenocopied this behavior, and induced EMT genes in prostate cancer cell lines. We purified exosomes from EDTA-treated, anticoagulated peripheral blood plasma of three T2D subjects and treated DU145 cells with equal numbers of exosomes ( $10^{9}$ in all cases) for two days, then isolated total RNA and assayed expression of the classical EMT genes vimentin (VIM), E-cadherin (CDH1), ANHAK and Snail (SNAI1) by RTPCR with TaqMan probes. As hypothesized, T2D plasma exosomes induced EMT genes compared to equal numbers of exosomes purified from plasma of ND subjects or media (complete growth media containing RPMI-1640 + 10\% FBS) control exosomes (Fig. 1A, Fig. S1). Having thus validated the DU145 cell responses by RT-PCR, we next analyzed these same total RNAs on a commercial array for well-established EMT genes [49] and observed that the T2D plasma exosomes induced a coherent, pro-EMT signature in the DU145 cells compared to ND controls (Fig. 1C). Of the significantly differentially expressed genes in the commercial microarray, the T2D exosomes most strongly repressed $C D H 1$, which encodes E-cadherin, as expected and as we have previously published for this gene associated with maintenance of the epithelial program and opposition to the mesenchymal program [49].

Ingenuity pathway analysis (IPA) of disease and function based on Fig. $\mathbf{1 C}$ revealed that plasma exosomes from T2D subjects strongly induced tumor cell aggressive features, such as cell spreading, protrusions, metastasis, cell motility and invasion compared to plasma exosomes from ND subjects (Fig. 2A), while pathways related to cell death by apoptosis or necrosis were 
downregulated by T2D exosomes, similar to what we have reported previously [49]. To confirm that T2D plasma exosomes also induce mesenchymal features (increased perimeter and elongation, and reduced circularity) as we previously reported [49], morphological parameters were analyzed using ImageJ. DU145 cells treated with T2D plasma exosomes showed increased elongation and perimeter, and decreased circularity, compared to cells treated with ND plasma exosomes and control cells treated with media-only exosomes (Fig. 2BC).

\subsection{Exosomes from plasma of T2D subjects induce PD-L1 expression in DU145 prostate} cancer cells

Gene signatures of EMT have been associated in several tumor types with immune infiltrates that express interferon-gamma (IFN- $\gamma$ )-induced genes, and correspondingly with elevated expression of immune checkpoint proteins, such as PD-L1 [56-58]. These associations prompted us to explore whether, in addition to inducing EMT networks, T2D plasma exosomes also upregulate expression of immune checkpoint genes compared to ND plasma exosomes. We found that plasma exosomes from T2D subjects did indeed upregulate genes that encode receptors important in cancer immunotherapy, including CD274 (Fig. 3A), which encodes PDL1, and CD155 (Fig. 3B), which encodes the poliovirus receptor and is associated with resistance to immune checkpoint therapy in several cancer types. Next, we analyzed the effect of T2D exosomes on DU145 cells, using a commercial array focused on genes involved in inflammation and cancer immune crosstalk. The T2D plasma exosomes induced a coherent, proinflammatory signature in DU145 cells compared to ND plasma exosomes (Fig. 3C). IPA analysis of the immune/inflammation microarray data from Fig. 3C showed that T2D plasma exosomes strongly upregulated major pathways associated with angiogenesis, immune dysfunction and tumor progression, compared to plasma exosomes from ND subjects (Fig. S2). 


\subsection{MicroRNA profiling of plasma exosomes}

Our previous studies on payload differences between exosomes released into conditioned media by mature adipocyte cultures from T2D vs ND subjects used mass spectrometry and proteomics to show that several proteins, particularly TSP5, were overrepresented in T2D exosomes [49]. Functional studies then showed that recombinant TSP5 alone was able to induce transcription of EMT gene signatures [49]. Therefore, we began with a similar proteomics-based approach to investigate peptide differences between T2D and ND exosomes purified from human plasma. However, proteomics profiling of three ND and three T2D exosome isolates revealed no distinct pattern of peptides that were differentially represented between the groups (Table S1). We considered an alternative hypothesis that miRNAs might be differentially expressed instead of proteins, and might encode functional activities that drive the observed EMT and immune checkpoint gene expression. We compared microRNA profiles of plasma exosomes from T2D and ND subjects by a human serum/plasma miRNA PCR array and found that the metabolic differences associate with a different miRNA signature. The five miRNAs that clustered as the most increased in T2D plasma exosomes compared to ND plasma exosomes were: miR-374a-5p, miR-93-5p, miR-28-3p, miR532-5p and miR375 (Fig. 4A). Other miRNAs showed reduced differential expression in T2D plasma exosomes compared to ND plasma exosomes. The five miRNAs that clustered as the most decreased were: miR-326, miR424-5p, miR-27a-3p, miR320b and miR320d (Fig. 4A).

We focused on miRNAs with known importance for cancer. Specifically, miR-374a-5p, which was highly elevated in T2D exosomes compared to ND controls, has been reported to contribute to liver cancer EMT and metastasis [59]. Additionally this miRNA is known to promote tumor progression by targeting $A R R B 1$ in triple negative breast cancer [60]. miR-93-5p, 
another miRNA that is highly expressed in T2D exosomes, has been identified as an oncogenic miRNA in a variety of human malignancies and involved in tumor angiogenesis [61]. Bioinformatics analysis suggests that miR-93-5p has important oncogenic functions in prostate cancer [62]. We also examined miRNAs that were significantly under-represented in T2D exosomes compared to ND controls. Among these, miR-326 has been reported to function as a tumor suppressor in human prostatic carcinoma by targeting Mucin1 [63] and miR326 has also been shown to inhibit melanoma progression by suppressing the AKT and ERK signaling pathways [64]. These reports provided a strong rationale to test individual miRNAs for function.

We obtained three of Fig. 4A miRNAs from commercial sources and treated DU145 cells with $25 \mathrm{nM}$ of each, for 2 days. Then total cellular RNA was isolated, cellular cDNA synthesized and fold-changes of SNAI1, CD274 and $C D H 1$ were compared to $A C T B$ as measured by RTPCR with TaqMan probes (Fig. 4BCD). We found that miR374a-5p upregulated CD274 (Fig. 4C) but not SNAI1 (Fig. 4B) and slightly downregulated CDH1 (Fig. 4D); miR-93-5p upregulated SNAI1 (Fig. 4B) but not CD274 (Fig. 4C) and did not affect CDH1 (Fig. 4D); and miR-424-5p downregulated CDH1 (Fig. 4D) but had no effect on SNAII (Fig. 4B) or CD274 (Fig. 4C). These results supported the overall hypothesis that T2D exosomal miRNAs are functionally active in tumor cell line models, when assayed as individual recombinant miRNAs.

We also performed a control experiment to prove that exosomal RNAs are taken up by DU145 cells. Exosome RNA was stained with RNA selective dye, then the stained exosomes were added to DU145 cells and visualized by fluorescence microscopy over several hours to track localization. Time course analysis showed that after 16 and 24 hours, the plasma exosomes RNA became concentrated in the nuclei (Fig. S3). Uptake reached a plateau by 16 hours. 


\subsection{RNA sequencing and Principal Component Analysis (PCA),}

Next, we investigated the global transcriptional changes in prostate cancer cell lines caused by T2D exosomes in comparison to the ND exosomes. RNA sequencing and Principal Component Analysis of all signals showed that total RNA transcripts from DU145 cells treated with exosomes isolated from plasma of ND subjects had similar global transcription patterns. The transcripts from cells treated with media-only control exosomes and ND plasma exosomes clustered together well. However, transcripts from DU145 cells treated with exosomes from T2D subjects were widely separated and unique. T2D samples 1 and 2 clearly separate from the other samples along PC1 (24\% of variance) and PC2 (14\% of variance), respectively, suggesting that there is significant biological variability with respect to treatment with the T2D exosomes (Fig. S4A). Our interpretation of this result is that ND plasma exosomes did not induce significant variation in genome-wide patterns of RNA expression compared to negative controls, but different T2D patient exosomes differ widely from ND controls, each in their own way. Furthermore, when T2D plasma exosomes are compared to ND, we identified a group of genes upregulated specifically by miR-103 (Fig. S4B), that has known functional roles in EMT and immune exhaustion.

We undertook IPA analysis of differential expression of all the genes in the datasets. Network analysis revealed that miR103a and SOX2-OT induce EMT and PD-L1 expression. MiR-103a and SOX2-OT were upregulated 28.8 and 21.8 times in DU145 cells treated with T2D exosomes compared to ND exosomes (Table S2, Fig. S4C). Interestingly, these two genes were downregulated (5 and 3.6-fold, respectively) in the ND exosome group compared to the mediaonly exosome control group (Table S2). The RNA seq datasets were further analyzed by IPA and the potential connections of dysregulated genes and cancer cell EMT and immune exhaustion through BET proteins were explored. IPA analysis revealed that miR103a and SOX2- 
OT induce Cav1 and VIM respectively, which ultimately promote EMT and PD-L1 expression through BRD4 (Fig. 5A). Additionally, RNA seq data showed that SNAI1 and CAV1 had an upregulated trend although it was not significant (Fig. S4B).

Our previous work has shown that the somatic BET bromodomain proteins BRD2, BRD3 and BRD4 play critical roles in transcriptional control of classical EMT genes $[53,65,66]$, as well as control of immune checkpoint genes, such as PDCD1 and CD274, which encode PD-1 and PD-L1, respectively. These findings have since been confirmed by others [67-69]. We therefore hypothesized here that the BET protein family functions as a central node in exosomedriven signal transduction, linking EMT to immune checkpoint function, and demonstrated here in prostate cancer cells. We have previously established in DU145 cells that low concentrations of the BRD4-selective PROTAC degrader MZ-1 (50 nM) are indeed selective to eliminate BRD4 protein while preserving BRD2 and BRD3, whereas high doses (400 nM) eliminate all three somatic BET proteins [51]. The small molecule JQ1 inhibits BET bromodomain activity through a different mechanism by competitively binding to the histone binding pocket of the bromodomain, and is not highly selective among BRD2, BRD3 and BRD4, thus we used it as a positive control for inhibition of all BET proteins, not just BRD4. We built on Fig. 4BC and measured transcription of SNAI1 and CD274 genes by RT-PCR. We found that MZ-1 at BRD4selective concentrations in DU145 cells inhibited expression of SNAII induced by T2D plasma exosomes, and the pan-BET inhibitor JQ1 was not able to reduce expression below the BRD4selective level (Fig. 5A). Interestingly, MZ-1 had the same inhibitory effect on CD274, but JQ1 was able to inhibit transcription to below baseline (Fig. 5A). We then verified by flow cytometry that PD-L1 protein expressed on the surface of treated DU145 cells was induced by T2D exosome treatment, and inhibited by MZ-1 or JQ1 (Fig. 5B). 


\section{Discussion}

Prostate cancer exhibits significant genomic and histologic heterogeneity that complicates prognostic assessment and clinical decision making. Disease can be indolent and localized, oligoclonal with non-overlapping mutational profiles among nearby clones [70], or aggressive with rapid progression and metastatic dissemination of a lethal clone [71]. A large subgroup of cases appears to be indolent at the early stage; it is important to resolve the indolent cases from aggressive cases that demand immediate treatment. Although serum level of Prostate Specific Antigen (PSA) has proven utility in combination with digital rectal examination for diagnostic screening [72, 73], PSA accuracy is suboptimal to understand cancer risks [74, 75]. Additional, noninvasive biomarkers have been explored for diagnostic, prognostic and therapeutic utility, including most recently circulating tumor DNA [31] and microRNAs (miRNA) [32]. In particular, miRNA has gained attention because, unlike other biomarkers, these factors may be functional in prostate cancer [33], and upon delivery to target tissues, are capable of reprogramming cell metabolism and fate to affect the course of progression, metastasis and therapeutic responses. Deeper understanding of miRNA mechanism and gene targets may suggest novel therapeutics or prognostic biomarkers [34] to understand progression risks.

Here, we took a new approach of exploring plasma exosomes for functional biomarkers that could prove useful for these prostate cancer patients. This report is the first to describe how plasma exosomes from subjects with Type 2 diabetes (i) drive pro-EMT transcriptional shifts and (ii) elevate immune checkpoint expression in human prostate cancer models. Surprisingly, we also found that plasma exosomes from ND controls showed activity to downregulate certain classical, pro-EMT genes, such as $S N A I 1$, and to upregulate certain classical anti-EMT genes, such as $C D H 1$, in prostate cancer cell lines (Fig. 1C). This observation suggests that ND status 
may encode chemopreventive factors that are packaged into plasma exosomes that circulate and may protect against prostate cancer progression.

Our initial approach to use differential proteomics analysis of the exosomes to identify peptides with significantly different abundance between T2D and ND exosomes recapitulated our previous approach, where we used proteomics analysis of exosomes purified from conditioned media of insulin-resistant vs insulin-sensitive mature adipocytes [49]. However, peptide differences between the two types of plasma exosomes were not significant enough to warrant deeper investigation. We turned instead to analysis of miRNAs to identify potential differences. Here, we found that commercial array kits were adequate to reveal interesting miRNA profiles.

To investigate this model, we compared the RNA seq data of DU145 cells treated with plasma exosomes from T2D and ND subjects. We found that the T2D exosomes upregulated a subset of genes that play critical roles in both EMT and immune exhaustion (Table 2). In order to illustrate the pathway, genes and their fold change values were imported to IPA. By using the path explorer feature of the software, the connections among upregulated genes, EMT genes and immune exhaustion genes were revealed (Fig. 5A). IPA output revealed that miR103 and SOX2OT stimulate $C A V 1$ and VIM respectively. Downstream in the pathway, BRD4 acts as the critical node, and activates $S N A I 1$ and CD274, which subsequently drive EMT and immune checkpoint expression. Other studies have proven that miR-103 promotes metastasis of colorectal cancer by targeting the metastasis suppressors DAPK and KLF4 [76]. Moreover, it has been reported that tumor derived miR-103 enhances cellular motility, invasion and EMT in esophageal squamous cell carcinoma [77], and oral squamous carcinoma cell [78]. In addition to miR-103, SOX2-OT was significantly upregulated in DU145 cells treated with T2D plasma exosomes. Published studies report that SOX2-OT facilitates proliferation and migration [79], invasion and metastasis 
of prostate cancer cells [80]. Taken together, these data suggest a new signaling map to link the pro-EMT effect of T2D plasma exosomes with PD-L1 expression through BRD4 (Fig. 6).

PD-L1 targeting is a major treatment strategy for several current cancer clinical trials of checkpoint inhibitors. The PDL1 findings may have clinical relevance related to utility of checkpoint inhibitors for treatment of advanced prostate cancer. Although checkpoint inhibitors have demonstrated significant efficacy in other advanced malignancies, the outcomes in advanced prostate cancer have been disappointing. Randomized phase III studies have failed to demonstrate an overall survival benefit in advanced prostate cancer [81, 82]. These studies, as well as several smaller studies with PD-1/PD-L1 inhibitors, did demonstrate clinical benefit in a small percentage of patients, yet there is ongoing need to identify markers to predict response or resistance. As we show here, Type 2 diabetes may work through plasma exosome crosstalk to increase the plasticity of prostate cancer primary cells, and induce immune exhaustion markers important for microenvironment interactions with tumor-infiltrating $\mathrm{T}$ cells. The consequences of this crosstalk for therapeutic agents, such as atezolizumab, are unknown and stratification by metabolic status in clinical trials using PD1/PDL1 inhibitors may provide further insights.

In conclusion, our findings show that T2D plasma exosomes induce prominent EMT and immune checkpoint markers in DU145 cells. Our study is the first report to unravel the oncogenic function of T2D plasma exosomes that could drive metastatic progression and treatment failure in castration-resistant prostate cancer. 
Figure Legends
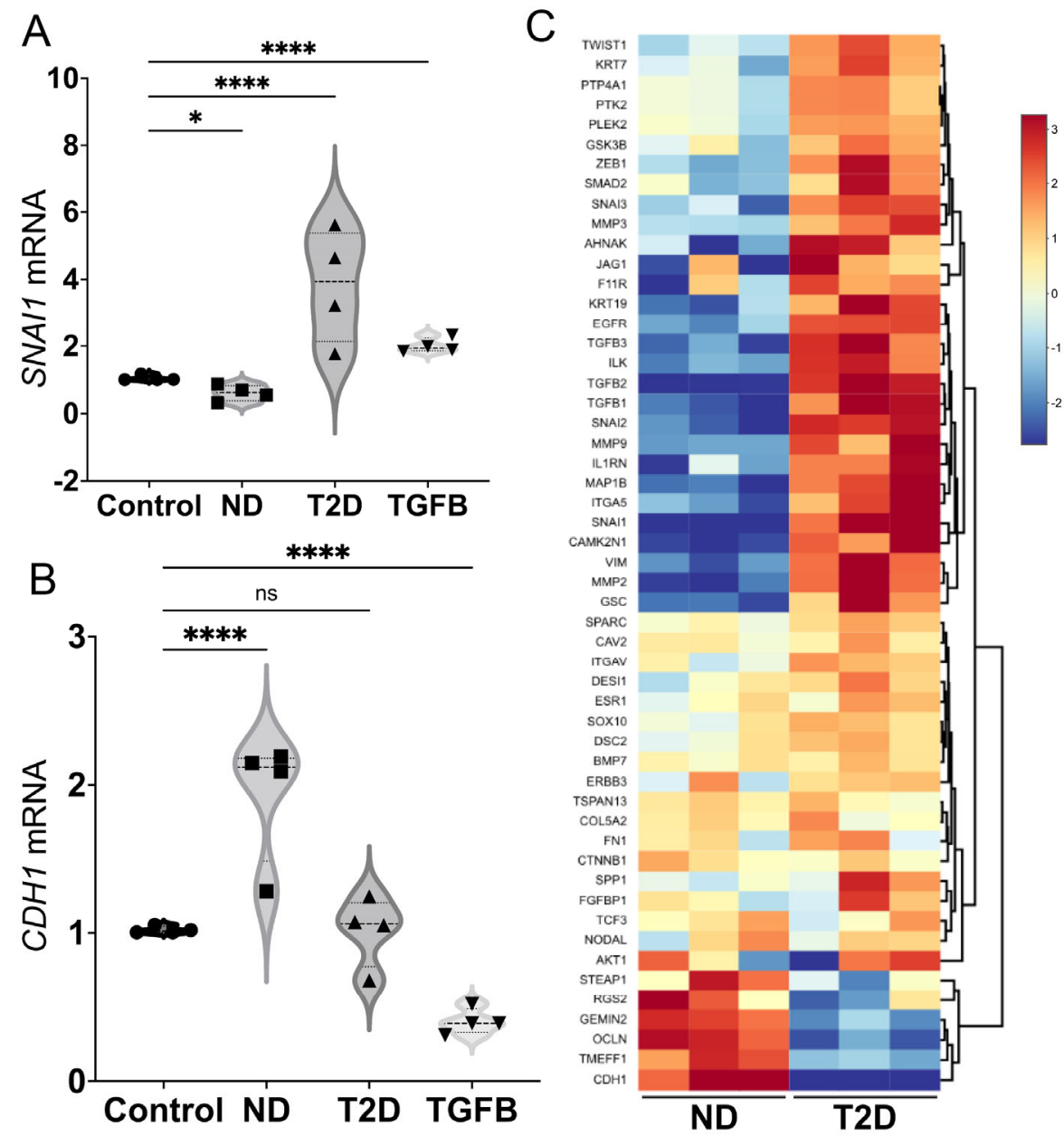

Fig. 1. Human plasma exosomes from T2D subjects induce EMT genes in prostate cancer cell lines. The DU145 human prostate cancer cell line was treated with either ND or T2D plasma exosomes $\left(10^{9}\right)$ for 2 days. (A) Plasma exosomes from four, independent T2D subjects $(\boldsymbol{\Delta})$ increased SNAII mRNA expression after normalizing to ACTB of the respective sample and compared to the control cells that were treated with growth media exosomes. $(\bullet)$. Exosomes from four, independent ND subjects ( $\boldsymbol{\square})$ did not increase $S N A I 1 \mathrm{mRNA}$ expression relative to $A C T B$ control. TGF- $\beta$ (TGFB, $5 \mathrm{ng} / \mathrm{mL}$ ) was used as a positive control ( $\boldsymbol{\nabla}$ ) for induction of proEMT gene transcription or repression of pro-MET gene transcription. (B) Plasma exosomes from T2D subjects did not induce $C D H 1$ expression. ND plasma exosomes increased $C D H 1$ mRNA expression. Data in A and B were obtained from four biological replicates of ND and T2D, and each biological replicate was conducted in three technical replicates, that are averaged in the graph. Data were analyzed by two-way ANOVA with statistical significance presented as: * $P=$ 0.0244; and $* * * *, P<0.0001$; $n s$, not significant. (C) Expression of selected EMT genes were analyzed by commercial PCR array. Relative expression of significantly differentially expressed genes in three independent, T2D exosome-treated samples was compared to three independent, ND exosome-treated samples. Equal numbers of exosomes $\left(10^{9}\right)$ from each sample were used. The heatmap of the PCR array result was calculated by hierarchical clustering. Scale bar (right) 
bioRxiv preprint doi: https://doi.org/10.1101/2022.02.01.478722; this version posted February 4, 2022. The copyright holder for this preprint (which was not certified by peer review) is the author/funder. All rights reserved. No reuse allowed without permission.

shows fold change, with red color to identify upregulation and blue color to identify downregulation. (ND, non-diabetic; T2D, Type 2 diabetic; TGFB, TGF- $\beta$ positive control) 

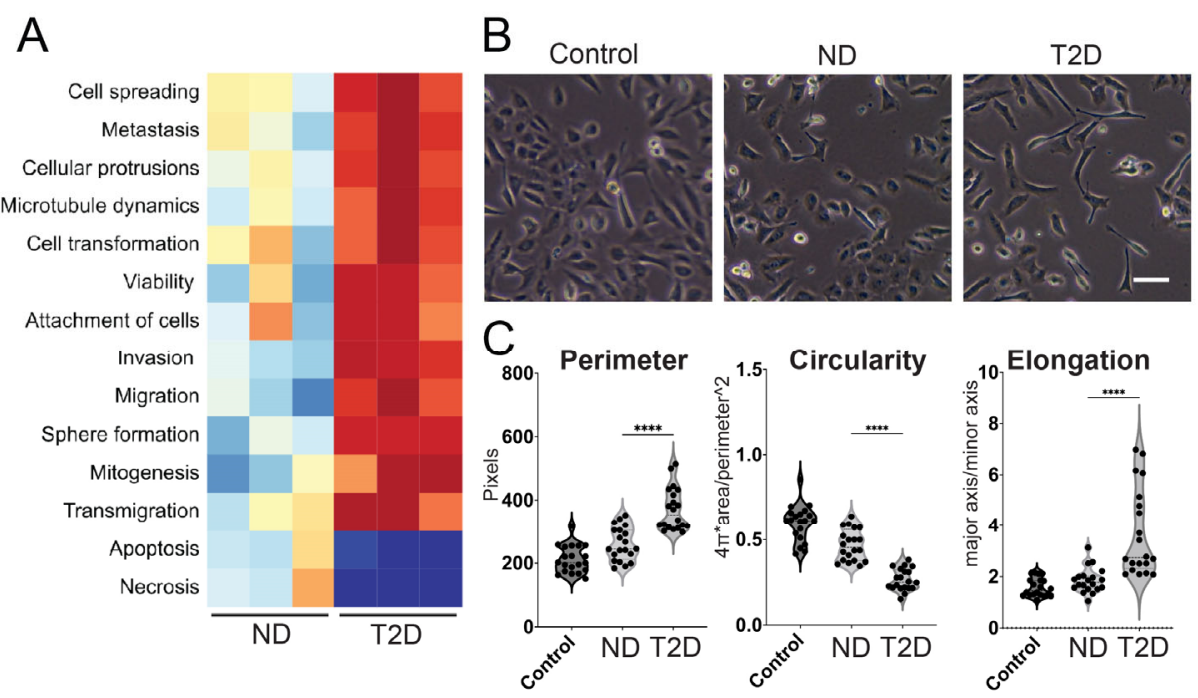

Fig. 2. Plasma exosomes from T2D subjects induce major features of tumor cell aggressive behavior. (A) Ingenuity pathway analysis (IPA) of disease and function based on Fig. 1C. IPA prediction shows that plasma exosomes from independent T2D subjects strongly induced tumor cell signatures associated with cancer aggressiveness compared to plasma exosomes from independent ND subjects. (B) Morphology of DU145 cells treated with equal number of plasma exosomes ND and T2D $\left(10^{9}\right)$ compared to the control cells treated with growth media (RPMI$1640+10 \%$ FBS) exosomes. One representative field of view is shown, out of 25 images collected for each of the three experimental conditions with three replicates. Scale bar, $30 \mu \mathrm{m}$. (C) Quantification of cell morphology, including cellular perimeter, circularity and elongation (a parameter that is converse to circularity) measured in images from B. Expression in each experimental was compared to control ( $\mathrm{n}=25$ cells each from $\mathrm{N}=3$ independent experiments). Data were analyzed by one-way ANOVA, with statistical significance presented as: ****, $\mathrm{p}<0.0001$. (ND, non-diabetic; T2D, Type 2 diabetic; Control, media-only exosomes) 


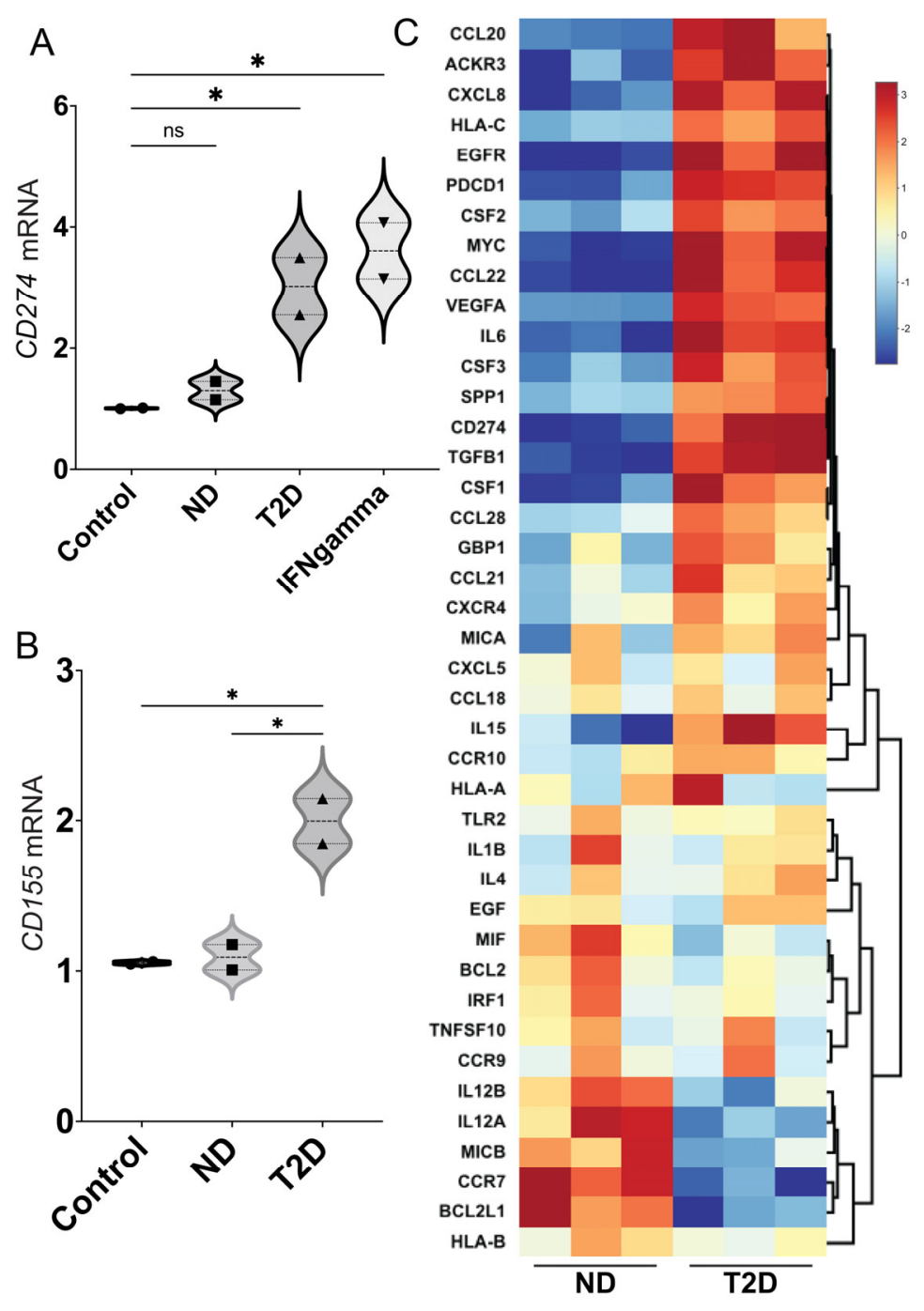

Fig. 3. T2D plasma exosomes upregulate genes that encode immune checkpoint ligands in prostate cancer cells. Plasma exosomes from T2D subjects $(\boldsymbol{\Delta})$ upregulated CD274 (A) and CD155 (B) mRNA expression in DU145 cells after 2 days, compared to ND control ( $\mathbf{(})$. Treatment with IFN- $\gamma(5 \mathrm{ng} / \mathrm{mL})$ was the positive control for $C D 274$ induction $(\boldsymbol{\nabla})$, as we previously published [53]. Treatment with growth media exosomes was the negative control $(\bullet)$. Data in A and B were obtained from two biological replicates of ND and T2D, and each biological replicate was conducted in three technical replicates. Data were analyzed by two-way ANOVA with statistical significance presented as: ${ }^{*}, P<0.05$; $n s$, not significant. (C) Hierarchical clustering of genes involved in inflammation and cancer immune crosstalk, analyzed by commercial PCR array. DU145 cells were treated with plasma exosomes from three T2D subjects or three ND subjects. Equal numbers of exosomes $\left(10^{9}\right)$ from each sample were used. The heatmap of the PCR array result was calculated by hierarchical clustering. Scale bar (right) shows fold change, with red color to identify upregulation and blue color to identify downregulation. (ND, non-diabetic; T2D, Type 2 diabetic; Control, media-only exosomes; IFNgamma, interferon- $\gamma$ ) 
A

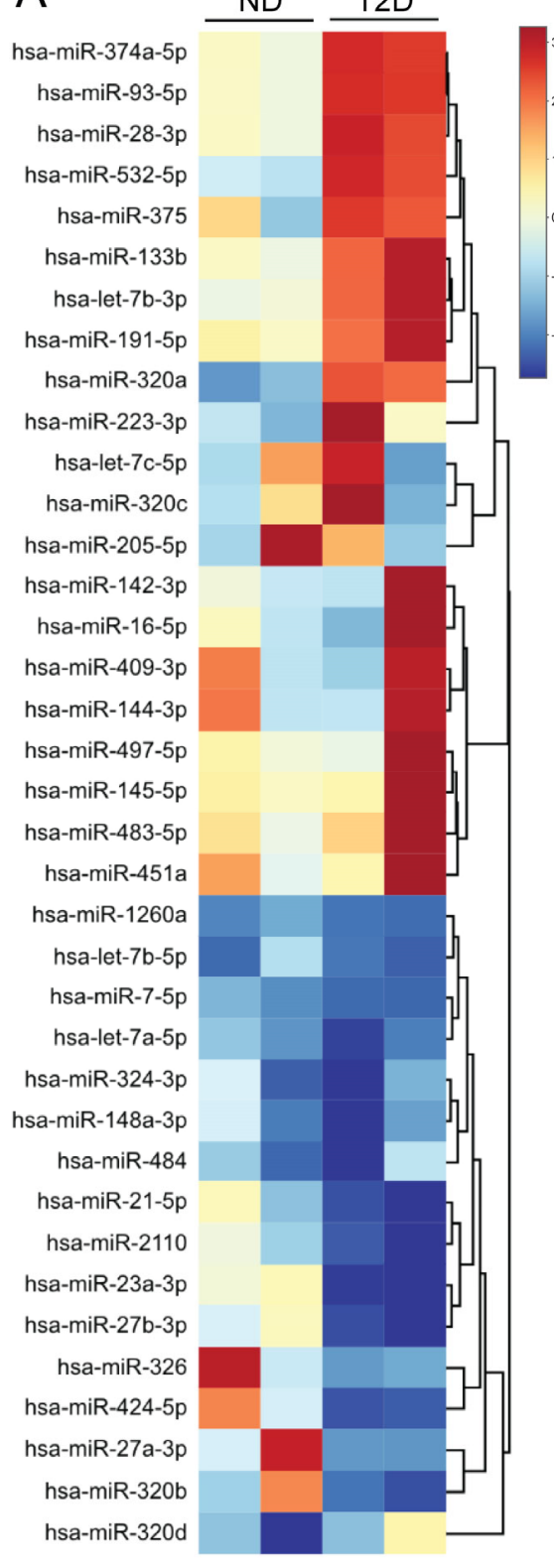

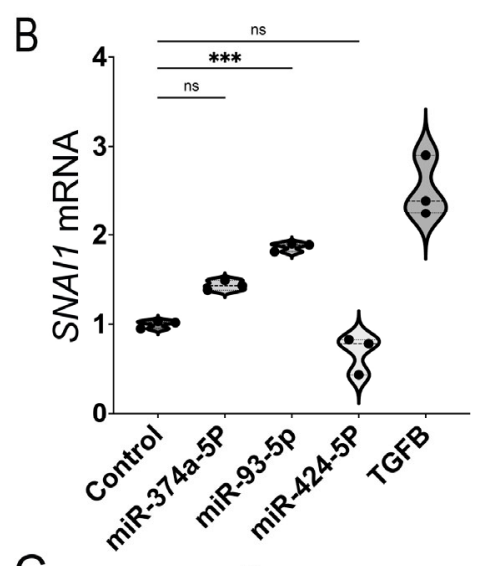

C
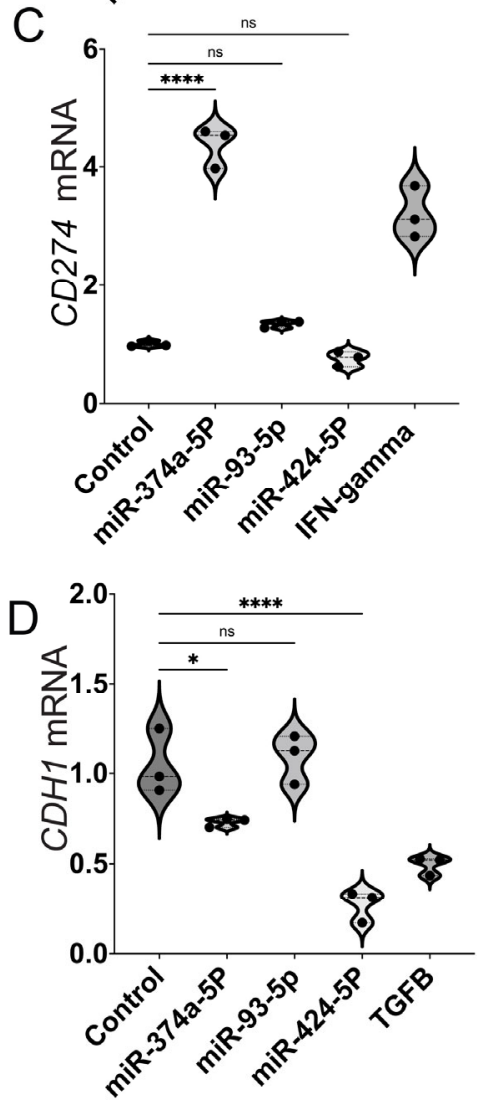

Fig. 4. T2D plasma exosomes have distinct microRNA profile compared to the ND plasma exosomes. (A) Heatmap of differentially expressed microRNAs in plasma exosomes of two T2D subjects compared to plasma exosomes of ND subjects. Total RNA was isolated from plasma exosomes of T2D and ND subjects, and miRNAs were profiled by a commercial PCR array. DU145 cells were transfected with individual miRNAs from (A) $(25 \mathrm{nM})$, selected based on their functional significance for EMT and immune exhaustion. The mRNA expression of SNAIl (B), $C D 274(\mathbf{C})$ and $C D H 1$ (D) was tested and expression relative to $\beta$-actin $(A C T B)$ is shown. Scale bar (A, right) shows fold change, with red color to identify upregulation and blue color to identify downregulation. Data were analyzed by one-way ANOVA with statistical significance presented as: *, $P<0.05$; $* * *, P<0.001$; ${ }^{* * * *}, P<0.0001$; $n s$, not significant. (Control, mediaonly exosomes; TGFB, TGF- $\beta$ positive control at $5 \mathrm{ng} / \mathrm{mL}$ for $S N A I 1$ induction; IFNgamma, interferon- $\gamma$ positive control at $5 \mathrm{ng} / \mathrm{mL}$ for $C D 274$ induction) 

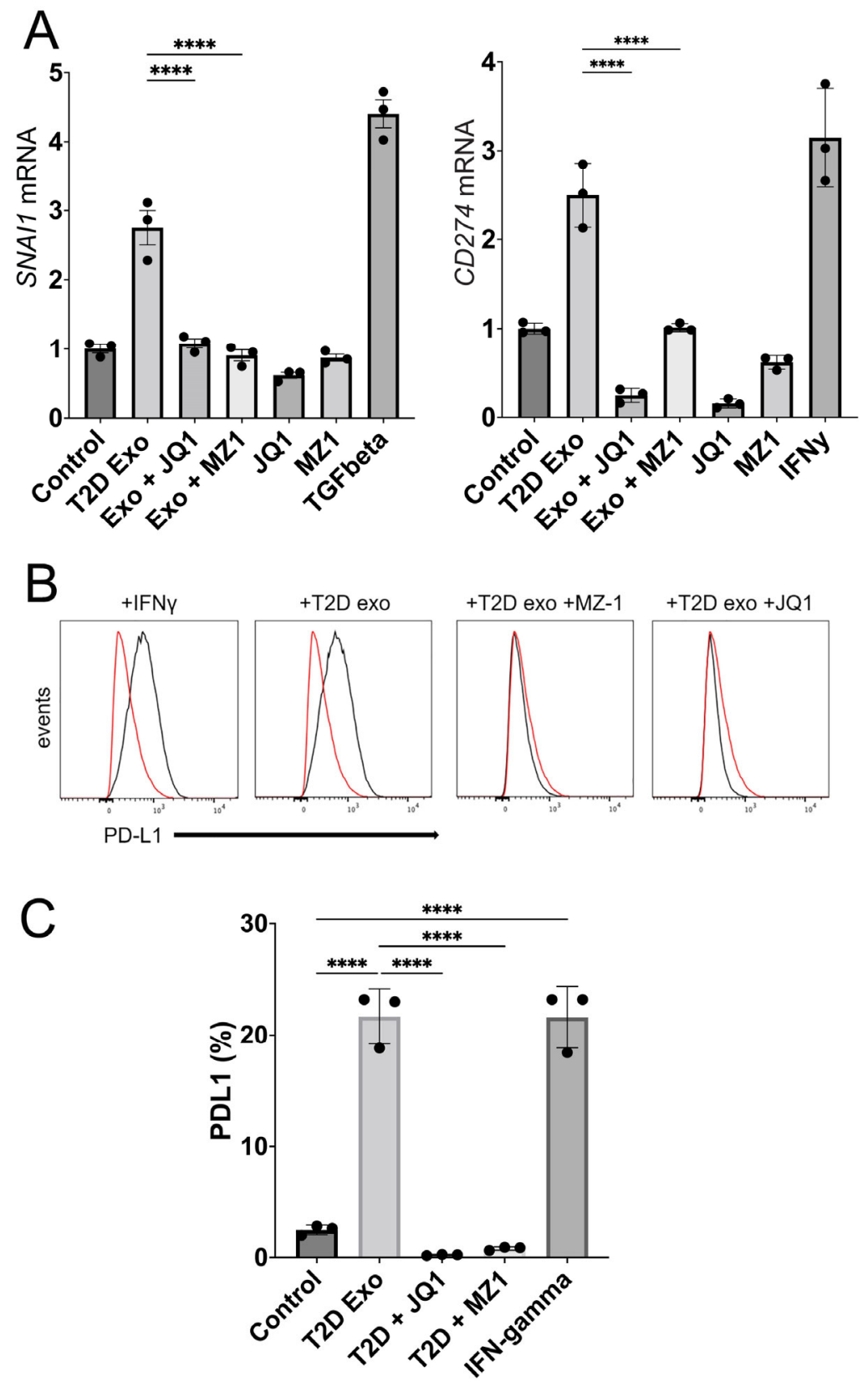

Fig. 5. T2D plasma exosomes require BRD4 to upregulate EMT genes and PD-L1

expression. (A) Expression of SNAI1 and CD274 genes in DU145 cells measured by RT-PCR of cellular RNA after exosome treatment. T2D exosomes $\left(10^{9} ;\right.$ T2D Exo $)$ were compared to mediaonly control exosomes (Control), or T2D Exo+JQ1 or MZ1. (JQ1 is a pan-BET inhibitor (400 $\mathrm{nM})$ and MZ-1 is a BRD4-selective degrader $(50 \mathrm{nM})$ ). (B) Expression of PD-L1 was measured by flow cytometry after treatments. One million events were analyzed by Flow-Jo and presented as overlaid histograms (control, red trace; experimental, black trace). (C) Flow cytometry data of B were quantified with PD-L1 as percent of parent population. The experiment was conducted in triplicate with differences between means represented as bar graphs. Data were analyzed by two-way ANOVA with statistical significance presented as: $* * * *, P<0.0001$ (TGFB, TGF- $\beta$ positive control at $5 \mathrm{ng} / \mathrm{mL}$ for $S N A I 1$ induction; IFNgamma, interferon- $\gamma$ positive control at 5 $\mathrm{ng} / \mathrm{mL}$ for $C D 274$ induction) 


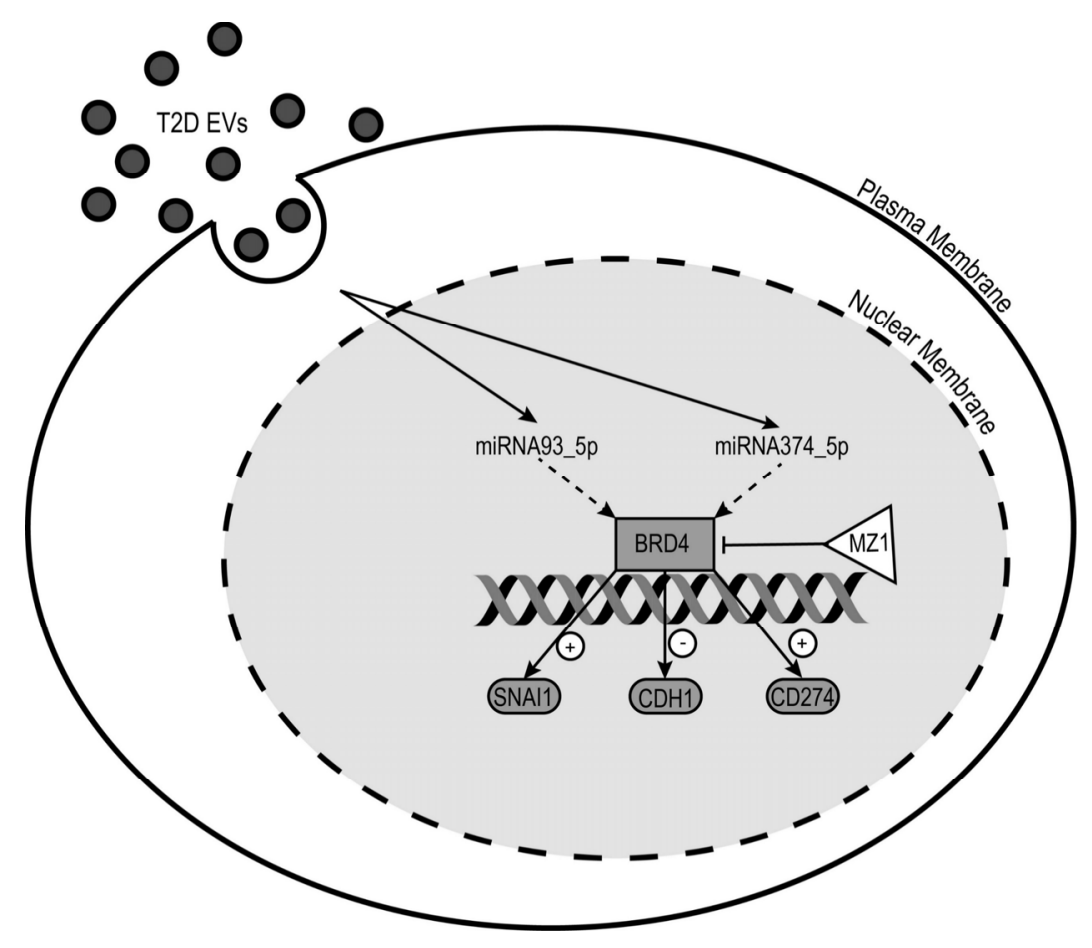

Fig. 6. Model for T2D exosome delivery of miRNAs that reprogram transcription of tumor cell genes important for cancer aggressiveness. Circulating exosomes in plasma arrive at the tumor cell surface where they are internalized and release their cargo of miRNAs. These miRNAs are trafficked to the nucleus where they reprogram signal transduction pathways. In this case, miR-93-5p and miR374a-5p are shown signaling through BRD4, which is an essential transcriptional co-regulator of genes important for tumor cell aggressiveness, SNAII, CDH1 and CD274. 

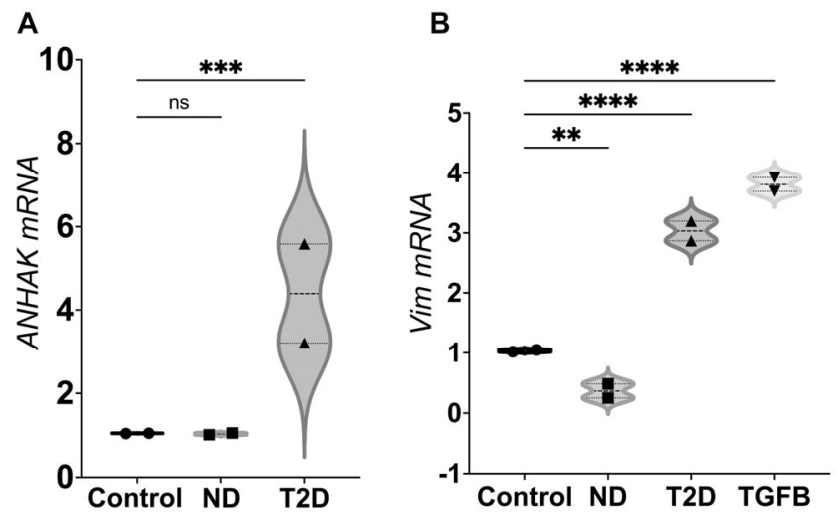

Supplemental Fig. S1. T2D exosome induction of selected EMT genes, determined by RTPCR. Human plasma exosomes from T2D subjects $(\boldsymbol{\Delta})$ induced representative EMT genes $A N H A K(\mathrm{~A})$ and VIMENTIN (Vim) (B) in DU145 cells, shown as fold-change relative to ACTB housekeeping gene $(\bullet)$. Responses for plasma exosomes from T2D subjects are compared to ND controls ( $\mathbf{\square})$. Data in A and B were obtained from two biological replicates of ND and T2D; each biological replicate was conducted in three technical replicates. Data were analyzed by twoway ANOVA, with statistical significance presented as: **, $\mathrm{p}<0.01 ; * * *, \mathrm{p}<0.001$; ****, $\mathrm{p}<0.0001$; $n s$, not significant. ND, non-diabetic; T2D, Type 2 diabetic; TGFB, TGF- $\beta$ positive control ( $\boldsymbol{\nabla})$. 


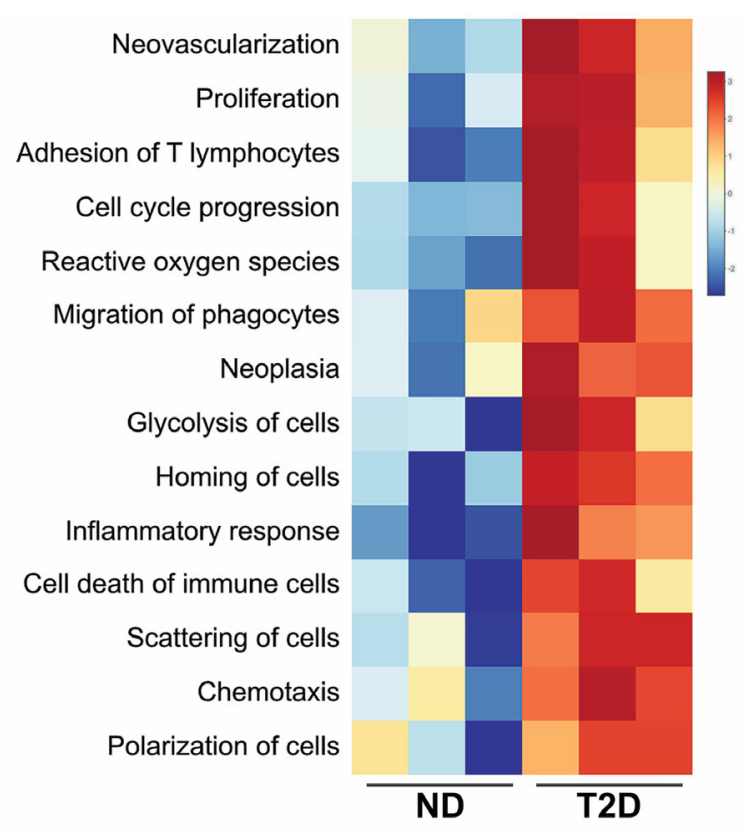

Supplemental Fig. S2. Ingenuity pathway analysis (IPA) of disease and function. IPA prediction based on Fig. 3C shows that plasma exosomes from T2D subjects induced major mechanisms associated with angiogenesis, immune dysfunction and tumor progression, compared to plasma exosomes from ND subjects. Three independent datasets for T2D are compared to three independent datasets for ND. Scale bar (right) shows fold change, with red color to identify upregulation and blue color to identify downregulation. (ND, non-diabetic; T2D, Type 2 diabetic) 


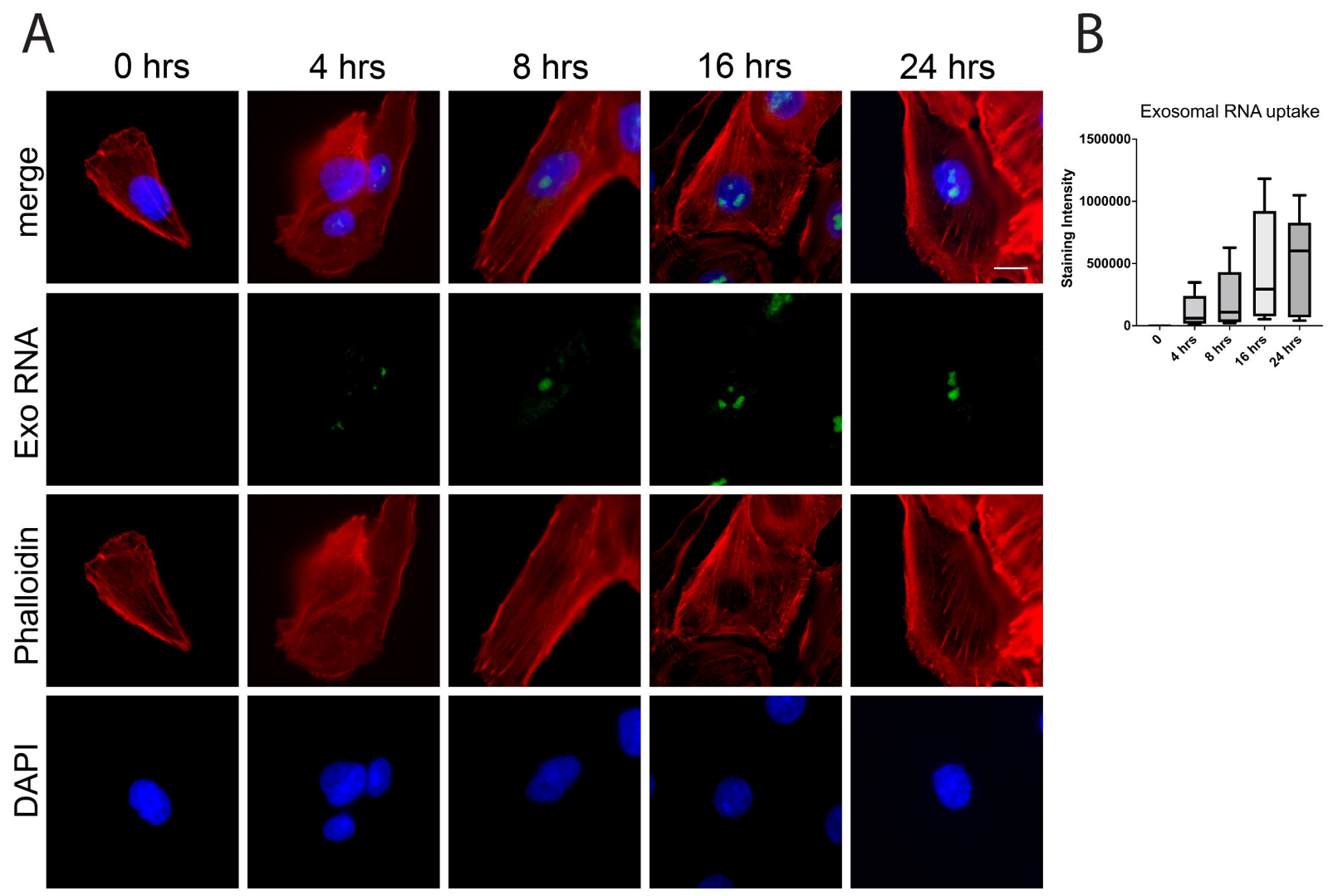

Supplemental Fig. S3. Time course of exosomal RNA uptake into DU145 cell nuclei. (A) Exosome RNA was stained with RNASelect (Exo RNA; Alexa Fluor ${ }^{\mathrm{TM}}-488$; green), then exosomes were washed and added to DU145 cells. Cells were imaged at 0, 4, 8, 16 and 24-hour time points. Filamentous actin (F-actin) was stained with Alexa Fluor ${ }^{\mathrm{TM}}$ Phalloidin probe 568nm (Phalloidin) and DNA was visualized by DAPI counterstain $(D A P I)$. One representative field of view is shown, out of 25 images collected for each of the three experimental conditions with three replicates. Scale bar, $10 \mu \mathrm{m}$. The mean intensity of stained RNA (Alexa Fluor ${ }^{\mathrm{TM}_{-} 488 \text { ) was }}$ quantified using ImageJ (B). 

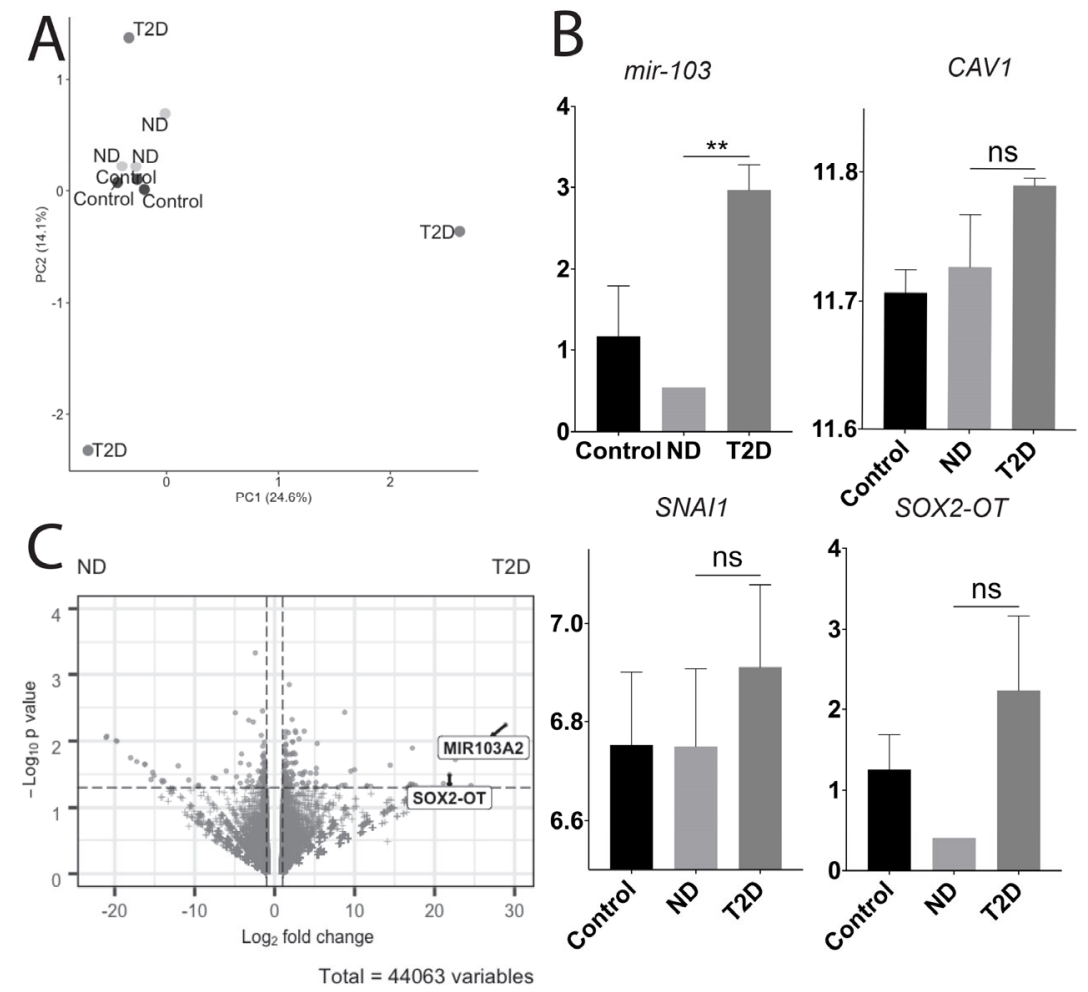

Supplemental Fig. S4. Genome-wide transcriptional analysis by RNA seq. (A) Plot of PC2 vs. PC1, computed across all genes in the DU145 samples. Plasma exosomes of T2D subjects had unique and different PCA values, while plasma exosomes of ND subjects had PCA values that were close to the media-only control values. (B) RNA seq expression of miR103a, SOX2OT, Cav1 and SNAI1 in DU145 cells treated with ND and T2D exosomes. The variance stabilizing transformed (VST) expression values for each gene were z-score-normalized to a mean of zero and standard deviation of 1 within all replicates of all samples. The Y axis shows the VST values. (C) Differential expression of genes unregulated in DU145 cells treated with T2D exosomes (right) vs. those of ND exosomes (left). Significantly differentially expressed genes were identified using a $p$ value cutoff of 0.05 and a fold change cutoff of 1 (dotted lines). Figure was generated using EnhancedVolcano package. 
bioRxiv preprint doi: https://doi.org/10.1101/2022.02.01.478722; this version posted February 4, 2022. The copyright holder for this preprint (which was not certified by peer review) is the author/funder. All rights reserved. No reuse allowed without permission.

\section{Table legends:}

Supplementary Table 1: Proteomics profile of plasma exosomes from 3 ND and 3 T2D subjects Supplementary Table 2: Fold change of T2D vs ND by RNA seq 
Competing interests: G.V.D. and N.J are inventors on U.S. patent 63/171,689 to use exosomes as a cancer diagnostic. The other authors declare that they have no conflict of interest. The funding agency played no role in the preparation of the manuscript or the decision to publish. Results and interpretation reported here do not necessarily represent the views of the NIH.

\section{Author Contributions}

Conception and design: G.V.D.

Methodology: N.J., M.K.

Acquisition of data: N.J., M.K, I.R.P., Y.Q., C.E.S.

Analysis and interpretation of data: N.J., M.K, I.R.P., Y.Q., C.E.S.

Writing, editing, and revision of manuscript: N.J., C.E.S., J.M., K.M., M.H., G.A.G., C.M.H, G.V.D.

Study supervision: G.V.D.

Funding: This work was supported by grants from the Cancer Systems Biology Consortium of the National Cancer Institute (GVD: U01CA182898, U01CA243004) and NCI Cancer Moonshot (GVD: R01CA222170), as well as with funding from the Shipley Prostate Cancer Research Center at Boston University (GVD).

Acknowledgements. We thank the Boston University-Boston Medical Center Flow Cytometry Core Facility, the Microarray and Sequencing Resource, and Cellular Imaging Core Facilities for technical assistance. 


\section{References}

[1] C. Steele, C. Thomas, Henley SJ, Massetti GM, Galuska DA, Agurs-Collins T, Puckett M, Richardson LC, Vital signs: trends in incidence of cancers associated with overweight and obesity-United States, 2014 (2005) 1052-1058.

[2] Centers for Disease Control and Prevention, National diabetes statistics report, 2020, Atlanta, GA: Centers for Disease Control and Prevention, US Department of Health and Human Services, (2020) 12-15.

[3] S. Yaturu, Obesity and type 2 diabetes, Journal of diabetes mellitus, 1 (2011) 79-95.

[4] S.T. Fleming, A. Rastogi, A. Dmitrienko, K.D. Johnson, A comprehensive prognostic index to predict survival based on multiple comorbidities: a focus on breast cancer, Medical care, (1999) 601-614.

[5] K.d.C.B. Ribeiro, L.P. Kowalski, M.d.R.D. De Oliveira, Perioperative complications, comorbidities, and survival in oral or oropharyngeal cancer, Archives of Otolaryngology-Head \& Neck Surgery, 129 (2003) 219-228.

[6] R. Rieker, E. Hammer, R. Eisele, E. Schmid, J. Högel, The impact of comorbidity on the overall survival and the cause of death in patients after colorectal cancer resection, Langenbeck's Archives of Surgery, 387 (2002) 72-76.

[7] C. Kastner, J. Armitage, A. Kimble, J. Rawal, P. Carter, S. Venn, The Charlson comorbidity score: a superior comorbidity assessment tool for the prostate cancer multidisciplinary meeting, Prostate cancer and prostatic diseases, 9 (2006) 270-274.

[8] A. Berglund, H. Garmo, C. Tishelman, L. Holmberg, P. Stattin, M. Lambe, Comorbidity, treatment and mortality: a population based cohort study of prostate cancer in PCBaSe Sweden, The Journal of urology, 185 (2011) 833-840.

[9] M. Extermann, Interaction between comorbidity and cancer, Cancer Control, 14 (2007) 1322.

[10] J. Coebergh, M. Janssen-Heijnen, P. Post, P. Razenberg, Serious co-morbidity among unselected cancer patients newly diagnosed in the southeastern part of The Netherlands in 19931996, Journal of clinical epidemiology, 52 (1999) 1131-1136.

[11] K.-L.C. Jen, K. Brogan, O.G. Washington, J.M. Flack, N.T. Artinian, Poor nutrient intake and high obese rate in an urban African American population with hypertension, Journal of the American College of Nutrition, 26 (2007) 57-65.

[12] R.B. Cadzow, T.J. Servoss, C.H. Fox, The health status of patients of a student-run free medical clinic in inner-city Buffalo, NY, The Journal of the American Board of Family Medicine, 20 (2007) 572-580.

[13] G.A. Nichols, M. McBurnie, L. Paul, J.E. Potter, S. McCann, K. Mayer, G. Melgar, S. D'Amato, J.E. DeVoe, Peer Reviewed: The High Prevalence of Diabetes in a Large Cohort of Patients Drawn From Safety Net Clinics, Preventing chronic disease, 13 (2016).

[14] H.K. Seligman, E.A. Jacobs, A. Lopez, U. Sarkar, J. Tschann, A. Fernandez, Food insecurity and hypoglycemia among safety net patients with diabetes, Archives of Internal Medicine, 171 (2011) 1204-1206.

[15] S.A. Berkowitz, X. Gao, K.L. Tucker, Food-insecure dietary patterns are associated with poor longitudinal glycemic control in diabetes: results from the Boston Puerto Rican Health study, Diabetes care, 37 (2014) 2587-2592.

[16] N.J. Vickers, Animal communication: when i'm calling you, will you answer too?, Current biology, 27 (2017) R713-R715. 
[17] C. Muller, Tumour-surrounding adipocytes are active players in breast cancer progression, Annales d'endocrinologie, Elsevier, 2013, pp. 108-110.

[18] L. Lore, H. An, L. Evelyne, V.B. Mieke, V. Jo, M. Dawn, B. Geert, V.D.B. Rudy, M. Cathérine, B. Marc, Secretome analysis of breast cancer-associated adipose tissue to identify paracrine regulators of breast cancer growth, Oncotarget, 8 (2017) 47239.

[19] C. Vaysse, J. Lømo, Ø. Garred, F. Fjeldheim, T. Lofteroed, E. Schlichting, A. McTiernan, H. Frydenberg, A. Husøy, S. Lundgren, Inflammation of mammary adipose tissue occurs in overweight and obese patients exhibiting early-stage breast cancer, NPJ breast cancer, 3 (2017) $1-10$.

[20] V. Laurent, A. Guérard, C. Mazerolles, S. Le Gonidec, A. Toulet, L. Nieto, F. Zaidi, B. Majed, D. Garandeau, Y. Socrier, Periprostatic adipocytes act as a driving force for prostate cancer progression in obesity, Nature communications, 7 (2016) 1-15.

[21] V. Laurent, A. Toulet, C. Attané, D. Milhas, S. Dauvillier, F. Zaidi, E. Clement, M. Cinato, S. Le Gonidec, A. Guérard, Periprostatic adipose tissue favors prostate cancer cell invasion in an obesity-dependent manner: role of oxidative stress, Molecular Cancer Research, 17 (2019) 821835.

[22] D. Estève, M. Roumiguié, C. Manceau, D. Milhas, C. Muller, Periprostatic adipose tissue: A heavy player in prostate cancer progression, Current Opinion in Endocrine and Metabolic Research, 10 (2020) 29-35.

[23] J. Hammarsten, B. Högstedt, Hyperinsulinaemia: a prospective risk factor for lethal clinical prostate cancer, European journal of cancer, 41 (2005) 2887-2895.

[24] J. Ma, H. Li, E. Giovannucci, L. Mucci, W. Qiu, P.L. Nguyen, J.M. Gaziano, M. Pollak, M.J. Stampfer, Prediagnostic body-mass index, plasma C-peptide concentration, and prostate cancer-specific mortality in men with prostate cancer: a long-term survival analysis, The lancet oncology, 9 (2008) 1039-1047.

[25] J.L. Wright, S.R. Plymate, M.P. Porter, J.L. Gore, D.W. Lin, E. Hu, S.B. Zeliadt, Hyperglycemia and prostate cancer recurrence in men treated for localized prostate cancer, Prostate cancer and prostatic diseases, 16 (2013) 204-208.

[26] T. Karantanos, S. Karanika, G. Gignac, Uncontrolled diabetes predicts poor response to novel antiandrogens, Endocrine-related cancer, 23 (2016) 691-698.

[27] L. Magura, R. Blanchard, B. Hope, J.R. Beal, G.G. Schwartz, A.E. Sahmoun, Hypercholesterolemia and prostate cancer: a hospital-based case-control study, Cancer Causes \& Control, 19 (2008) 1259-1266.

[28] E. Kheterpal, J.D. Sammon, M. Diaz, A. Bhandari, Q.-D. Trinh, N. Pokala, P. Sharma, M. Menon, P.K. Agarwal, Effect of metabolic syndrome on pathologic features of prostate cancer, Urologic Oncology: Seminars and Original Investigations, Elsevier, 2013, pp. 1054-1059. [29] J. Flanagan, P.K. Gray, N. Hahn, J. Hayes, L. Myers, C. Carney-Doebbeling, C. Sweeney, Presence of the metabolic syndrome is associated with shorter time to castration-resistant prostate cancer, Annals of Oncology, 22 (2011) 801-807.

[30] I.-C. Yu, H.-Y. Lin, J.D. Sparks, S. Yeh, C. Chang, Androgen receptor roles in insulin resistance and obesity in males: the linkage of androgen-deprivation therapy to metabolic syndrome, Diabetes, 63 (2014) 3180-3188.

[31] A.W. Wyatt, M. Annala, R. Aggarwal, K. Beja, F. Feng, J. Youngren, A. Foye, P. Lloyd, M. Nykter, T.M. Beer, Concordance of circulating tumor DNA and matched metastatic tissue biopsy in prostate cancer, JNCI: Journal of the National Cancer Institute, 109 (2017).

[32] R. Kanwal, A.R. Plaga, X. Liu, G.C. Shukla, S. Gupta, MicroRNAs in prostate cancer:

Functional role as biomarkers, Cancer Letters, 407 (2017) 9-20. 
[33] A.M. Aghdam, A. Amiri, R. Salarinia, A. Masoudifar, F. Ghasemi, H. Mirzaei, MicroRNAs as diagnostic, prognostic, and therapeutic biomarkers in prostate cancer, Critical Reviews ${ }^{\mathrm{TM}}$ in Eukaryotic Gene Expression, 29 (2019).

[34] A. Watahiki, R.J. Macfarlane, M.E. Gleave, F. Crea, Y. Wang, C.D. Helgason, K.N. Chi, Plasma miRNAs as biomarkers to identify patients with castration-resistant metastatic prostate cancer, International journal of molecular sciences, 14 (2013) 7757-7770.

[35] G. Rabinowits, C. Gerçel-Taylor, J.M. Day, D.D. Taylor, G.H. Kloecker, Exosomal microRNA: a diagnostic marker for lung cancer, Clinical lung cancer, 10 (2009) 42-46. [36] A. Michael, S.D. Bajracharya, P.S. Yuen, H. Zhou, R.A. Star, G.G. Illei, I. Alevizos, Exosomes from human saliva as a source of microRNA biomarkers, Oral diseases, 16 (2010) 3438.

[37] J. Skog, T. Würdinger, S. van Rijn, D. Meijer, L. Gainche, Glioblastoma microvesicles transport RNA and proteins that promote tumour growth and provide diagnostic biomarkers. Nat Cell Biol10 (12): 1470-6.[10.1038/ncb1800] Chapter 1 of tumor-derived exosomes as diagnostic biomarkers of ovarian cancer, Gynecol Oncol, 110 (2008) 13-21.

[38] R. Nedaeinia, M. Manian, M. Jazayeri, M. Ranjbar, R. Salehi, M. Sharifi, F. Mohaghegh, M. Goli, S. Jahednia, A. Avan, Circulating exosomes and exosomal microRNAs as biomarkers in gastrointestinal cancer, Cancer gene therapy, 24 (2017) 48-56.

[39] N. Kosaka, H. Iguchi, T. Ochiya, Circulating microRNA in body fluid: a new potential biomarker for cancer diagnosis and prognosis, Cancer science, 101 (2010) 2087-2092.

[40] T. Matsumura, K. Sugimachi, H. Iinuma, Y. Takahashi, J. Kurashige, G. Sawada, M. Ueda, R. Uchi, H. Ueo, Y. Takano, Exosomal microRNA in serum is a novel biomarker of recurrence in human colorectal cancer, British journal of cancer, 113 (2015) 275-281.

[41] S.J. Freedland, W.J. Aronson, Examining the relationship between obesity and prostate cancer, Reviews in Urology, 6 (2004) 73.

[42] K. Di Sebastiano, J. Pinthus, W. Duivenvoorden, M. Mourtzakis, Glucose impairments and insulin resistance in prostate cancer: The role of obesity, nutrition and exercise, Obesity Reviews, 19 (2018) 1008-1016.

[43] F. Nik-Ahd, L.E. Howard, A.T. Eisenberg, W.J. Aronson, M.K. Terris, M.R. Cooperberg, C.L. Amling, C.J. Kane, S.J. Freedland, Poorly controlled diabetes increases the risk of metastases and castration-resistant prostate cancer in men undergoing radical prostatectomy: Results from the SEARCH database, Cancer, 125 (2019) 2861-2867.

[44] S. Kelkar, T. Oyekunle, A. Eisenberg, L. Howard, W.J. Aronson, C.J. Kane, C.L. Amling, M.R. Cooperberg, Z. Klaassen, M.K. Terris, Diabetes and Prostate Cancer Outcomes in Obese and Nonobese Men After Radical Prostatectomy, JNCI Cancer Spectrum, 5 (2021) pkab023. [45] M.F. Sona, S.-K. Myung, K. Park, G. Jargalsaikhan, Type 1 diabetes mellitus and risk of cancer: a meta-analysis of observational studies, Japanese journal of clinical oncology, 48 (2018) 426-433.

[46] R. Peila, T.E. Rohan, Diabetes, glycated hemoglobin, and risk of cancer in the UK biobank study, Cancer Epidemiology and Prevention Biomarkers, 29 (2020) 1107-1119.

[47] A.J. Burton, K.M. Tilling, J.M. Holly, F.C. Hamdy, M.-A.E. Rowlands, J.L. Donovan, R.M. Martin, Metabolic imbalance and prostate cancer progression, International journal of molecular epidemiology and genetics, 1 (2010) 248.

[48] A.J. Klil-Drori, L. Azoulay, M.N. Pollak, Cancer, obesity, diabetes, and antidiabetic drugs: is the fog clearing?, Nature reviews Clinical oncology, 14 (2017) 85-99.

[49] N. Jafari, M. Kolla, T. Meshulam, J.S. Shafran, Y. Qiu, A.N. Casey, I.R. Pompa, C.S. Ennis, C.S. Mazzeo, N. Rabhi, S.R. Farmer, G.V. Denis, Adipocyte-derived exosomes may promote breast cancer progression in type 2 diabetes, Science Signaling, 14 (2021) eabj2807. 
[50] B. Zhang, Y. Yang, X. Shi, W. Liao, M. Chen, A.S.-L. Cheng, H. Yan, C. Fang, S. Zhang, $\mathrm{G}$. Xu, Proton pump inhibitor pantoprazole abrogates adriamycin-resistant gastric cancer cell invasiveness via suppression of $\mathrm{Akt} / \mathrm{GSK}-\beta / \beta$-catenin signaling and epithelial-mesenchymal transition, Cancer letters, 356 (2015) 704-712.

[51] J.S. Shafran, G.P. Andrieu, B. Györffy, G.V. Denis, BRD4 regulates metastatic potential of castration-resistant prostate cancer through AHNAK, Molecular Cancer Research, 17 (2019) 1627-1638.

[52] J.S. Shafran, N. Jafari, A.N. Casey, B. Győrffy, G.V. Denis, BRD4 regulates key transcription factors that drive epithelial-mesenchymal transition in castration-resistant prostate cancer, Prostate cancer and prostatic diseases, 24 (2021) 268-277.

[53] G.P. Andrieu, J.S. Shafran, C.L. Smith, A.C. Belkina, A.N. Casey, N. Jafari, G.V. Denis, BET protein targeting suppresses the PD-1/PD-L1 pathway in triple-negative breast cancer and elicits anti-tumor immune response, Cancer letters, 465 (2019) 45-58.

[54] A. Dobin, C.A. Davis, F. Schlesinger, J. Drenkow, C. Zaleski, S. Jha, P. Batut, M. Chaisson, T.R. Gingeras, STAR: ultrafast universal RNA-seq aligner, Bioinformatics, 29 (2013) 15-21. [55] M.I. Love, W. Huber, S. Anders, Moderated estimation of fold change and dispersion for RNA-seq data with DESeq2, Genome biology, 15 (2014) 1-21.

[56] Y. Lou, L. Diao, E.R.P. Cuentas, W.L. Denning, L. Chen, Y.H. Fan, L.A. Byers, J. Wang, V.A. Papadimitrakopoulou, C. Behrens, Epithelial-mesenchymal transition is associated with a distinct tumor microenvironment including elevation of inflammatory signals and multiple immune checkpoints in lung adenocarcinoma, Clinical Cancer Research, 22 (2016) 3630-3642. [57] J.C. Thompson, W.-T. Hwang, C. Davis, C. Deshpande, S. Jeffries, Y. Rajpurohit, V. Krishna, D. Smirnov, R. Verona, M.V. Lorenzi, Gene signatures of tumor inflammation and epithelial-to-mesenchymal transition (EMT) predict responses to immune checkpoint blockade in lung cancer with high accuracy, Lung Cancer, 139 (2020) 1-8.

[58] M. Taki, K. Abiko, M. Ukita, R. Murakami, K. Yamanoi, K. Yamaguchi, J. Hamanishi, T. Baba, N. Matsumura, M. Mandai, Tumor Immune Microenvironment during EpithelialMesenchymal Transition, Clinical Cancer Research, 27 (2021) 4669-4679.

[59] Q. Lin, C.-R. Zhou, M.-J. Bai, D. Zhu, J.-W. Chen, H.-F. Wang, M.-A. Li, C. Wu, Z.-R. Li, M.-S. Huang, Exosome-mediated miRNA delivery promotes liver cancer EMT and metastasis, American journal of translational research, 12 (2020) 1080.

[60] D. Son, Y. Kim, S. Lim, H.-G. Kang, D.-H. Kim, J.W. Park, W. Cheong, H.K. Kong, W. Han, W.-Y. Park, miR-374a-5p promotes tumor progression by targeting ARRB1 in triple negative breast cancer, Cancer letters, 454 (2019) 224-233.

[61] L. Liang, L. Zhao, Y. Zan, Q. Zhu, J. Ren, X. Zhao, MiR-93-5p enhances growth and angiogenesis capacity of HUVECs by down-regulating EPLIN, Oncotarget, 8 (2017) 107033. [62] Y. Yang, B. Jia, X. Zhao, Y. Wang, W. Ye, miR-93-5p may be an important oncogene in prostate cancer by bioinformatics analysis, Journal of cellular biochemistry, 120 (2019) 1046310483.

[63] X. Liang, Z. Li, Q. Men, Y. Li, H. Li, T. Chong, miR-326 functions as a tumor suppressor in human prostatic carcinoma by targeting Mucin1, Biomedicine \& Pharmacotherapy, 108 (2018) 574-583.

[64] K. Kang, J. Zhang, X. Zhang, Z. Chen, MicroRNA-326 inhibits melanoma progression by targeting KRAS and suppressing the AKT and ERK signalling pathways, Oncology reports, 39 (2018) 401-410.

[65] G. Andrieu, A.H. Tran, K.J. Strissel, G.V. Denis, BRD4 regulates breast cancer dissemination through Jagged1/Notch1 signaling, Cancer research, 76 (2016) 6555-6567. 
[66] G.P. Andrieu, G.V. Denis, BET proteins exhibit transcriptional and functional opposition in the epithelial-to-mesenchymal transition, Molecular Cancer Research, 16 (2018) 580-586. [67] S.J. Hogg, S.J. Vervoort, S. Deswal, C.J. Ott, J. Li, L.A. Cluse, P.A. Beavis, P.K. Darcy, B.P. Martin, A. Spencer, BET-bromodomain inhibitors engage the host immune system and regulate expression of the immune checkpoint ligand PD-L1, Cell reports, 18 (2017) 2162-2174. [68] X. Wang, Y. Zhou, Y. Peng, T. Huang, F. Xia, T. Yang, Q. Duan, W. Zhang, Bromodomain-containing protein 4 contributes to renal fibrosis through the induction of epithelial-mesenchymal transition, Experimental cell research, 383 (2019) 111507.

[69] X. Jing, S. Shao, Y. Zhang, A. Luo, L. Zhao, L. Zhang, S. Gu, X. Zhao, BRD4 inhibition suppresses PD-L1 expression in triple-negative breast cancer, Experimental cell research, 392 (2020) 112034.

[70] M.C. Haffner, W. Zwart, M.P. Roudier, L.D. True, W.G. Nelson, J.I. Epstein, A.M. De Marzo, P.S. Nelson, S. Yegnasubramanian, Genomic and phenotypic heterogeneity in prostate cancer, Nature Reviews Urology, 18 (2021) 79-92.

[71] O. Sartor, J.S. de Bono, Metastatic prostate cancer, New England Journal of Medicine, 378 (2018) 645-657.

[72] W.J. Catalona, D.S. Smith, T.L. Ratliff, K.M. Dodds, D.E. Coplen, J.J. Yuan, J.A. Petros, G.L. Andriole, Measurement of prostate-specific antigen in serum as a screening test for prostate cancer, New England Journal of Medicine, 324 (1991) 1156-1161.

[73] R. Etzioni, A. Tsodikov, A. Mariotto, A. Szabo, S. Falcon, J. Wegelin, D. Ditommaso, K. Karnofski, R. Gulati, D.F. Penson, Quantifying the role of PSA screening in the US prostate cancer mortality decline, Cancer Causes \& Control, 19 (2008) 175-181.

[74] B. Djavan, A. Zlotta, M. Remzi, K. Ghawidel, A. Basharkhah, C.C. SCHULMAN, M. MARBERGER, Optimal predictors of prostate cancer on repeat prostate biopsy: a prospective study of 1,051 men, The Journal of urology, 163 (2000) 1144-1149.

[75] I.M. Thompson, D.K. Pauler, P.J. Goodman, C.M. Tangen, M.S. Lucia, H.L. Parnes, L.M. Minasian, L.G. Ford, S.M. Lippman, E.D. Crawford, Prevalence of prostate cancer among men with a prostate-specific antigen level $\leq 4.0 \mathrm{ng}$ per milliliter, New England Journal of Medicine, 350 (2004) 2239-2246.

[76] H.Y. Chen, Y.M. Lin, H.C. Chung, Y.D. Lang, C.J. Lin, J. Huang, W.C. Wang, F.M. Lin, Z. Chen, H.D. Huang, miR-103/107 promote metastasis of colorectal cancer by targeting the metastasis suppressors DAPK and KLF4, Cancer research, 72 (2012) 3631-3641.

[77] D. Gao, B. Hou, D. Zhou, Q. Liu, K. Zhang, X. Lu, J. Zhang, H. Zheng, J. Dai, Tumorderived exosomal miR-103a-2-5p facilitates esophageal squamous cell carcinoma cell proliferation and migration, Eur. Rev. Med. Pharmacol. Sci, 24 (2020) 6097-6110.

[78] X. Liu, Y. Cao, Y. Zhang, H. Zhou, H. Li, Regulatory effect of MiR103 on proliferation, EMT and invasion of oral squamous carcinoma cell through SALL4, Eur. Rev. Med. Pharmacol. Sci, 23 (2019) 9931-9938.

[79] Q. Wo, D. Zhang, L. Hu, J. Lyu, F. Xiang, W. Zheng, J. Shou, X. Qi, Long noncoding RNA SOX2-OT facilitates prostate cancer cell proliferation and migration via miR-369-3p/CFL2 axis, Biochemical and biophysical research communications, 520 (2019) 586-593.

[80] X. Song, H. Wang, J. Wu, Y. Sun, Long Noncoding RNA SOX2-OT Knockdown Inhibits

Proliferation and Metastasis of Prostate Cancer Cells Through Modulating the miR-452-

5p/HMGB3 Axis and Inactivating Wnt/ $\beta$-Catenin Pathway, Cancer biotherapy \& radiopharmaceuticals, 35 (2020) 682-695.

[81] E.D. Kwon, C.G. Drake, H.I. Scher, K. Fizazi, A. Bossi, A.J. Van den Eertwegh, M.

Krainer, N. Houede, R. Santos, H. Mahammedi, Ipilimumab versus placebo after radiotherapy in patients with metastatic castration-resistant prostate cancer that had progressed after docetaxel 
chemotherapy (CA184-043): a multicentre, randomised, double-blind, phase 3 trial, The lancet oncology, 15 (2014) 700-712.

[82] T. Powles, K.C. Yuen, S. Gillessen, E.E. Kadel, D. Rathkopf, N. Matsubara, C.G. Drake, K. Fizazi, J.M. Piulats, P.J. Wysocki, Atezolizumab with enzalutamide versus enzalutamide alone in metastatic castration-resistant prostate cancer: a randomized phase 3 trial, Nature medicine, (2022) 1-10. 